$\mathbb{T}$ periodica polytechnica

Mechanical Engineering

$55 / 2$ (2011) $9 1 \longdiv { 9 4 }$

doi: 10.3311/pp.me.2011-2.05

web: http://www.pp.bme.hu/me

(c) Periodica Polytechnica 2011

RESEARCH ARTICLE

\section{Application of bioethanol in gas turbines}

Viktor Józsa

Received 2011-11-31

\section{Abstract}

To reduce our dependence on primer energy resources, renewable fuels are appearing in an increasing number of fields. Environment has a major effect on theoperating conditions of a gas turbine. Thermal efficiency and power loss occurs, due to higher ambient temperature in summers. There are certain methods to reduce gas turbine inlet temperature, e.g. water evaporation, adsorption and mechanical cooling. Ours is the following: inject bioethanol into the inlet air, which will evaporate, so it reduces inlet temperature. Emission and efficiency measurements were taken to evaluate this method. The Department's Capstone C330 gas turbine use airblast atomizers for liquid fuel evaporation. Air, as auxiliary medium was replaced with ethanol-water compound steam,which can be easily produced with waste heat of the gas turbine. A test pad was built to examine an isolated fuel nozzle in operation. Pictures and high frame rate videos were taken for visual evaluation. This information helped us to fine-tune our system, and then optimal operating conditions were determined.

\section{Keywords}

gas turbine · renewable fuel · bioethanol · evaporation

\section{Acknowledgement}

Thanks to Krisztián Sztankó, Attila Kun-Balog and Sándor Könczöl for theoretical and technical support, employees of laboratory, who helped me so much in assembling the measurement devices. This project was supported by ÁMFT TÁMOP4.2.1/B-09/1/KMR-[2010-0002] and also supported by the grant TÁMOP-4.2.2.B-10/1-2010-0009.

\section{Viktor Józsa}

BME Department of Energy Engineering, H-1111 Budapest, Bertalan Lajos street. 4-6, Hungary

\section{Introduction}

In the alcohol industry pre- and post-distillates are secondary products while making different kind of beverages. These types of alcohols are considered hazardous, so disposing is required by the law. This unavoidable process has a relatively high cost. The alcohol content of these distillates varies, so heating value can even be quite low, and as a result it is rarely worth using them, as a main fuel of a heat engine [3].

Utilization is recommended from many viewpoints. It is renewable fuel, so power generating application is highly supported all around the world. Moreover, disposal costs can be saved by the industry as well. Lastly, it will reduce the company's need for energy if used locally.

It is known from Carnot cycle the maximum efficiency of a heat engine is determined by the average temperature of heat addition and heat rejection. In this case, we focus on reducing the inlet temperature of a micro gas turbine, which then leads to efficiency increase, because the average temperature of heat outflow will be lower.

Applying water evaporation in dry, hot places like a desert is a widely used method to increase efficiency over a long period of time. It is the cheapest way to reduce inlet temperature of the gas turbine in these regions. However, in the European climate humidity is quite often $70-80 \%$ or even more, so this method cannot be effectively used. Other solutions (e.g. adsorption and mechanical cooling) to reduce inlet temperature are relatively expensive. [1]. Alcohol which is injected directly into inlet air with nearly $100 \%$ humidity can evaporate, because of partial pressure. So this solution is universal, because it is almost independent from humidity and relatively cheap. The application area can be anywhere, but it's worth from the equator up to the temperate climate zone.

Measurements were taken in the BME, laboratory of Department of Energy Engineering, using a Capstone C330 natural gas fueled micro gas turbine.

\section{Measurement system}

The aim of measurement was to show efficiency increasing influence of ethanol evaporation in different ambient temperatures 
and also to analyze emission changes.

Capstone C330's maximum electrical performance is $30 \mathrm{~kW}$; so demanded power was set to $20 \mathrm{~kW}$ during examinations. Nominal thermal efficiency is $26 \pm 2 \%$, because of recuperator. This micro gas turbine can be used in mini cogeneration, if the exhausted gas is driven through an external heat exchanger.

The demanded temperature of the inlet air was set by an air preparation unit. The first sampled temperature was compressor inlet by the micro gas turbine's inner measurement system.

Ethanol was injected into air with a regular fuel atomizer, used in Otto engines. Control of this was achieved by a function generator by setting duty cycle. Ethanol was pumped by a hydraulic supply system.

Measurement data of the gas turbine and emission analyzer was recorded by 2 PCs.

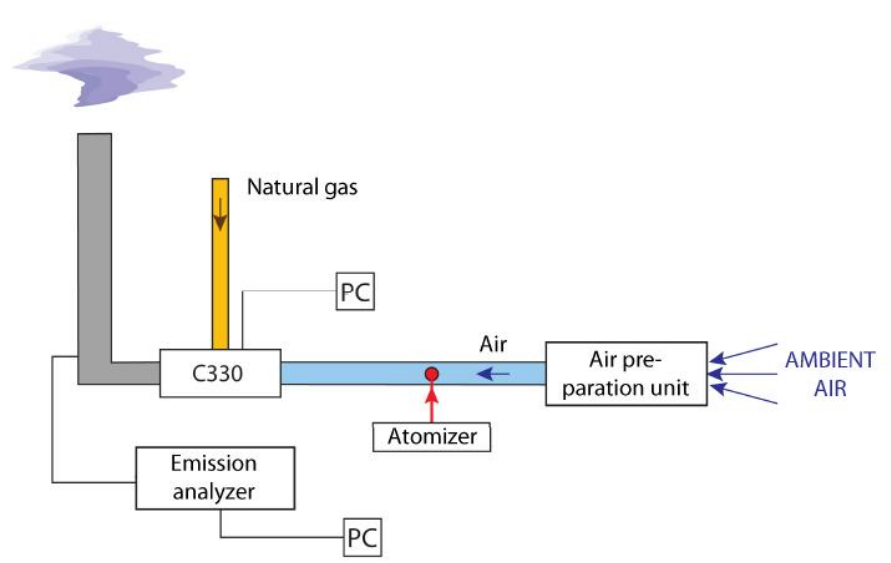

Fig. 1. Measurement system.

\section{Measurements}

First of all a measurement was made to show to what extent efficiency decreases with temperature. The inlet temperature was set in every measured point by the air preparation unit. Fig. 2]contains the result.

As Fig. 2 shows inlet temperature has just a minor influence on thermal efficiency. However we know in the energy industry a tiny change in operation can cause high financial consequences on a yearly basis. So every possibility to increase efficiency must be considered.

In the next step, a constant inlet temperature was set in the preheater, and then series of measurements were focused on the influence of ethanol evaporation on the total efficiency. Ethanol mass flow was varied from zero up to $10 \%$ of flash concentration for safety reasons. The cooling effect on the inlet temperature was present, as expected, but if we add the heat content of the injected ethanol to the heat content of natural gas efficiency change will be the following: It is possible that unburned ethanol caused reduction in the efficiency. The more ethanol injected to inlet air; the more leaves gas turbine without burning. Because of 10 air to fuel ratio, large amount of inlet air, which is already mixed with evaporated ethanol, could not burn, so left with flue gas. From a power generating viewpoint, using
Influence of compressor inlet temeprature on efficiency

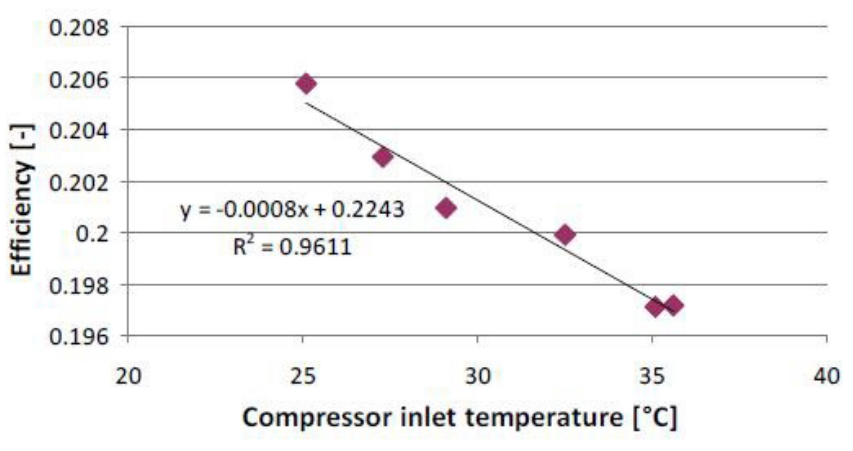

Fig. 2. Influence of inlet temperature on efficiency.

\section{Influence of evaporation on efficiency}

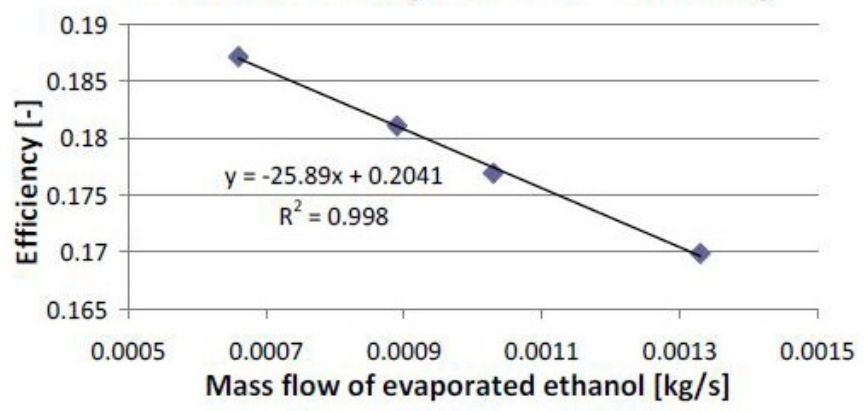

Fig. 3. Influence of injection on efficiency.

\section{$\mathrm{UHC}$ and $\mathrm{CO}$ emission growth}

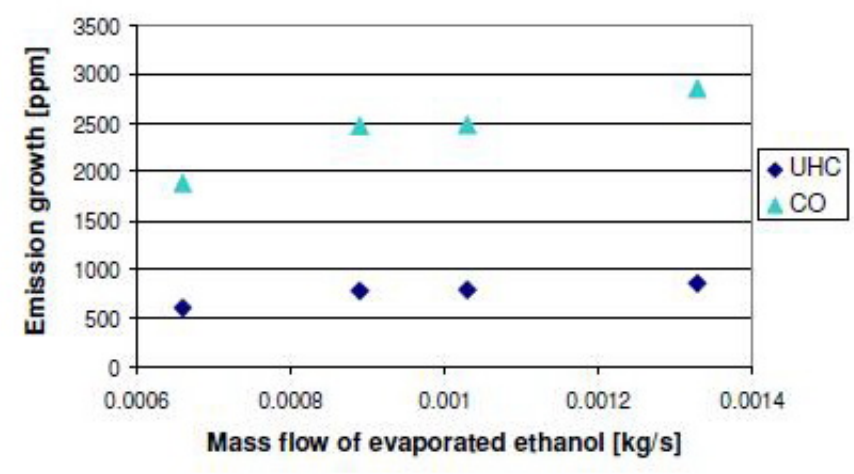

NOx emission reduction

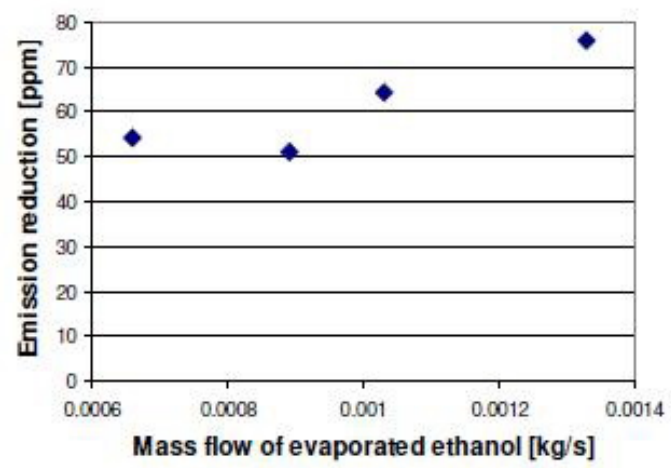

Fig. 4. Result of emission measurement. 
alcohol-water compound as fuel is more efficient. This measurement took a long time, so the changing of ambient conditions must be considered. One was taken with ethanol injection, and then another without it, when the alcohol completely left the system. So comparing these results can be done with lesser measurement error, because of lesser changes in ambient temperature.

The emission measurement showed us that it is a certain ethanol that couldn't be burned completely, which lead to efficiency loss. UHC (unburned hydrocarbon) and $C O$ (carbon monoxide) content of exhausted gas is exceeded for so many times current standard's limits. Ethanol evaporation had no influence on $\mathrm{CO} 2$ emission. However, there was positive change in the $N O x$ (nitrogen oxides) content, because with injection it's halved, even if the ethanol mass flow was low.

Due to very high emission bioethanol cannot be used in a gas turbine this way, since evaporated alcohol left the system without burning. Highly efficient usage and lower emission can be achieved, if ethanol is injected directly into the combustion chamber. Because of varying composition and heating value it is not appropriate to mix this with the main fuel and burn them together in order to protect the fuel system. This compound would absorb much heat for warming up and phase transition and lowers the flame temperature, if it's injected directly in liquid state. With waste heat required energy for these processes can be covered, and the steam of bioethanol-water compound will not absorb significant amount of heat inside the combustor.

\section{Isolated fuel nozzle test pad}

Capstone C330 micro gas turbine has 3 airblast atomizers. An isolated fuel nozzle was examined, but air was replaced with steam of the water-alcohol compound as an auxiliary medium for atomization. Required heat can be covered by waste heat in gas turbine application.

This test pad operated in atmospheric pressure instead of the pressure chamber with 3.5 bar (this is the C330's typical pressure after the compressor stage) absolute pressure inside. It is known from combustion techniques increase in pressure results wider stable burning limits. So recommended operating conditions will be fine for gas turbine application as well [2].

The fuel nozzle has partly radial, partly tangential inlet orifices. So inlet air enters the nozzle with swirl to improve premixing. Ambient air was conveyed by a centrifugal fan. There was a built in rotameter to measure volume flow rate. Demanded combustion air temperature was set by a controllable air preheater to simulate C330 gas turbine combustor environments.

Fuel was supplied by a voltage controlled gear pump. The fuel nozzle was designed to operate with diesel fuel and kerosene.

The most complex part of test pad is evaporating medium generator system. A gear pump conveyed water-ethanol compound also. Superheated steam was produced in two steps. First, a preheater increases the temperature of the liquid mixture up to

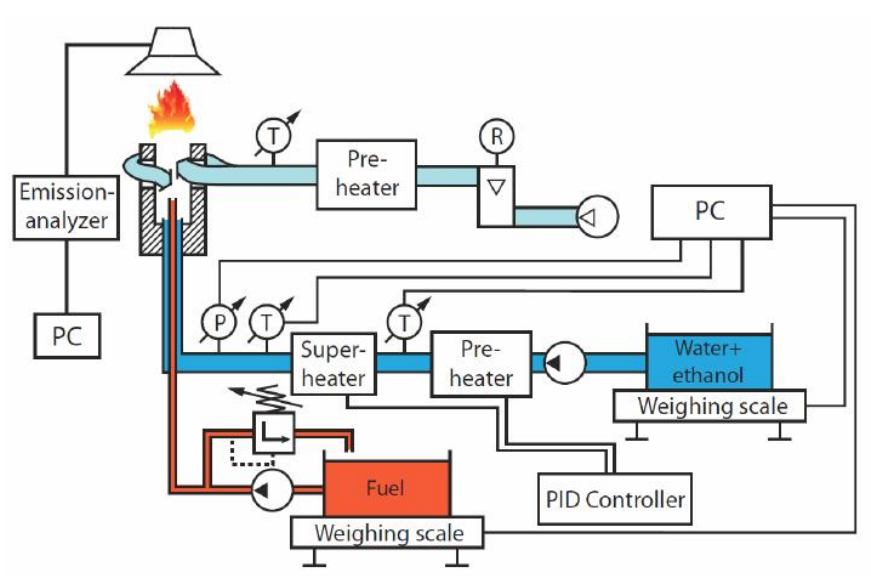

Fig. 5. Test pad (T: resistance thermometer, P: pressure transducer, R: rotameter)

almost boiling point with a maximum performance of $1.5 \mathrm{~kW}$. Second, a serially connected second heating cartridge with the same performance produces steam and also heating it up to $180^{\circ} \mathrm{C}$. Because alcohol and water have different specific heat and boiling point, these physical properties must be well known to stay in a stable operating region. A PID controller made by HAGA Automation was responsible for setting and holding the desired temperatures in heating cartridges.

A steel tube was paced concentrically with the fuel nozzle for flame stabilization. A Bunsen burner was used for ignition.

Fuel and water-ethanol tanks were placed on weighing scales. The actual weight data was recorded in every 30 seconds, so consumption can be calculated from it's change over time.

\section{Analysis of flame}

During measurement pictures and high speed videos were recorded for visual $t$ evaluation. The temperature of preheater, superheater and pressure of evaporation were also saved. Recording time of photos and measured data were synchronized. As the examination shows superheating temperature has just a minor influence on evaporation quality and flame development. However, pressure is clearly the most important property of evaporation steam.

Pictures from inhomogeneous flames show that there were too large droplets, which could not evaporate completely until flame front. Fig. 2 and Fig. 3 in Fig. 6 are good examples of insufficient droplet production, because of the presence of yellow stripes. Critical pressure ratio was present, while pictures 1 and 4 were taken. So the velocity of the outflow was the local speed of sound. In picture 5 there was higher fuel mass flow, but the evaporation quality is still flawless, and there were not any stripes present (trajectory of burning, not evaporated fuel droplets), even with relatively long (1/30) shutter speed. 
Fig. 6. Flames.
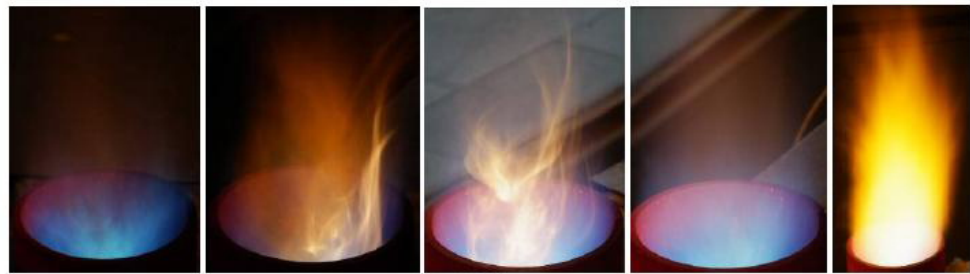

Tab. 1. Data of Fig.6

\begin{tabular}{llllll}
\hline $\begin{array}{l}\text { (pictures from left to } \\
\text { right) }\end{array}$ & Picture & Picture & Picture & Picture & Picture \\
\hline $\begin{array}{l}\text { Shutter speed [s] } \\
\begin{array}{l}\text { Evaporation pressure } \\
\text { [bar] }\end{array}\end{array}$ & 2,1 & 2 & 3 & 4 & 5 \\
\hline
\end{tabular}

\section{Conclusion}

Inlet air temperature can be reduced by ethanol injection, so thermal efficiency increases. Because of 10 air to fuel ratio and maximum $850{ }^{\circ} \mathrm{C}$ turbine inlet temperature, ethanol could not burn completely, so emission was unacceptably high. There are no standards which allow these outstanding concentration values.NOX content of flue gas was the only one which decreased significantly in every case. This examination is worth to repeating in a larger power generating gas turbine with low air to fuel ratio and higher turbine inlet temperature for emission analysis.

To not exceed emission standards ethanol should be applied with high efficiency, so it is appropriate to inject bioethanol directly into the combustor. Capstone $\mathrm{C} 330$ micro gas turbine uses airblast atomizers. A test pad was built to examine the operation of an isolated fuel nozzle with alcohol-water compound steam instead of air auxiliary medium. Required heat for steam generation was covered by two heating cartridges, each one had $1.5 \mathrm{~kW}$ heating performance. During gas turbine application, waste heat will be used for boiling and superheating. Evaluation of measured data and pictures showed steam temperature has no influence on flame quality. Critical or more pressure ratio is needed for fine fuel evaporation. Even with more fuel mass flow, and then operational maximum, the breakup of the fuel jet was perfect. Relatively large droplets, which leave yellow stripes in photos, cannot be found in the pictures, even with lower shutter speed, which indicates large droplets.

\section{References}

1 Boyce PM, Gas Turbine Engineering Handbook, Second Edition, Texas: Gulf Publishing Company, 1995.

2 Lefebvre AH, Ballal DR, Gas Turbine Combustion, Third Edition, CRC Press.

3 Sztankó K, Kun-Balog A, Alcohol based fuel utilization in micro gas turbine. Heat engines and environmental protection, Balatonfüred, Hungary, 2011, pp. 159-164. 